\title{
Developments on Phased Array for Low-Cost, High Frequency Applications
}

\author{
Przemyslaw Gorski ${ }^{1}$, Maria Carolina Vigano ${ }^{2}$, Daniel Llorens del $\mathrm{Rio}^{3}$ \\ ${ }^{1}$ ViaSat Antenna Systems SA, Lausanne, Switzerland, przemyslaw.gorski@viasat.com \\ ${ }^{2}$ ViaSat Antenna Systems SA, Lausanne, Switzerland, maria.vigano@viasat.com \\ ${ }^{3}$ ViaSat Antenna Systems SA, Lausanne, Switzerland, daniel.1lorens@viasat.com
}

\begin{abstract}
This work presents the design and implementation of a phased array antenna for reconfigurable 5G backhaul network links. In order to minimize costs, the antenna has been developed using low cost $\mathrm{RO4003}$ substrates and off the shelf electronic components. The antenna operates in $\mathrm{Ka}$ band $(19 \mathrm{GHz})$ with scan range down to $50^{\circ}$.
\end{abstract}

Index Terms-antenna, phased array, beam forming network, scanning.

\section{INTRODUCTION}

Modern telecommunication systems demand ever increasing capacity to accommodate fast growing smartphone traffic, including telepresence and support for remote processing. The backhaul network must be able to accommodate this capacity without compromising robustness [1]. The bandwidth resources in $\mathrm{L}, \mathrm{S}$ and $\mathrm{Ku}$ band are limited, but the required capacity is available in the millimeter wave frequency and above. Communication in these bands suffer from higher signal loss due to atmospheric absorption and rain, requiring a high antenna gain. Furthermore, some of the bands of interest are not for exclusive terrestrial use, but must be shared with the satellite services. Therefore the ability to control interference can enable the use of additional bandwidth for terrestrial use. Reconfigurable antennas that allow multibeam and multicast operation can multiply the connectivity of the backhaul network and make it resilient against failures or temporary traffic spikes.

A highly directive, steerable beam antenna is not only desirable in modern communications but is required in numerous systems such as in radar, satellite communication or space exploration. In the earliest beam steering antennas [2] mechanical pointing was used to mitigate unwanted signals. In the following decades other solutions for steering beam antennas have been developed, such as direct radiating arrays, focal array fed reflectors, parasitic array antennas, array-fed lens antennas, switched beam antennas, traveling wave antennas, retro-directive antennas, and metamaterial antennas [3].

Steering the beam with a mechanical solution is attractive from a cost perspective, but results in a bulky configuration, slow steering speed, and dubious reliability. Array fed reflectors and integrated lens antennas are faster and more reliable, but are still bulky and difficult to integrate with existing systems, and may suffer from other defects such as limited scan range or limited nulling capabilities. Parasitic steering is a compact solution but it lacks of the capability to have high steer resolution especially for high frequency [4].

Direct radiating arrays offer high resolution and many degrees of freedom for beam shaping and interference cancellation, but require complex control units and possibly downconverters. Up until recently only custom components were available, especially at Ka-band. Even now, the chips available off-the-shelf (discrete components such as phase shifters, attenuators and amplifiers) are expensive and have high insertion losses, although the cost can be reduced with larger production volumes. The cost and losses can be further reduced through an ad hoc chip integrating all the needed function although this is only cost-effective if a product with large manufacturing quantities is envisaged.. Such a 'core chip' is more compact than an assembly of discrete components as well as enabling wider scan and lower board prices.

TABLE I. shows the cost of production of microwave chips (MMICs) in 2005. For a given process, price depends on chip area. The estimated size of a chip including phase shifter and variable gain amplifier operating in $\mathrm{Ka}$ band should be below $2 \times 2 \mathrm{~mm}$, which results in a price below $2 \$$.

TABLE I. CHIP COST AGAINST SIZE [5].

\begin{tabular}{|c|c|c|c|}
\hline Chip size $\left(\mathrm{mm}^{2}\right)$ & $\begin{array}{c}\text { Typical yield } \\
(\%)\end{array}$ & $\begin{array}{c}\text { Working } \\
\text { circuits per 6\% } \\
\text { wafer }\end{array}$ & $\begin{array}{c}\text { Bare chip cost } \\
(\$) \text { at \$5k per } \\
\text { wafer }\end{array}$ \\
\hline $1 \times 1$ & 80 & 12800 & 0.4 \\
\hline $2 \times 2$ & 70 & 2800 & 1.8 \\
\hline $5 \times 5$ & 45 & 288 & 17 \\
\hline $7 \times 7$ & 30 & 98 & 51 \\
\hline $10 \times 10$ & 20 & 32 & 156 \\
\hline
\end{tabular}

Printed technology with active MMIC is a solution that perfectly matches the low-cost requirements.

\section{PHASED ARRAY ANTENNA FOR BACKHAUL NETWORK}

In an efficient backhaul network each base station may need to communicate with multiple nodes (base stations) whilst avoiding interferences and supporting fast data rates. This requisite leads to many antenna requirements such as the capability for multibeam operation, multicast radiation patterns, and high gain. The possibility of steering the beam(s) at least in one plane (depending on the local network environment) is also desired e.g. in rush hours in order to 
rebalance the traffic among the base stations; or during a storm when some links might go down. According to these requirements, we present a transmit phased array prototype operating at $19 \mathrm{GHz}$, capable of generating two simultaneous beams. With respect to a classic antenna for satellite communication on the same frequency band, the adopted polarization is single linear because the antenna is used for terrestrial fixed link where circular polarization is not required. To connect the antenna to the base station infrastructure a downconverter unit has been added. The antenna can scan a beam in one plane with a directivity above $25 \mathrm{~dB}$. This value can be easily increased by adding extra radiating elements.

\section{A. Antenna Element}

The exploded view of proposed antenna element is depicted on Fig. 1. A single patch radiator is driven by the symmetric stripline through an H-shaped slot. Four through via-holes around the slot suppress the parallel plate mode. To reduce the cost, a RO4003 substrate has been used for the patch and the feeding lines. The feed line incorporates a transformer to improve the impedance match of the antenna element.

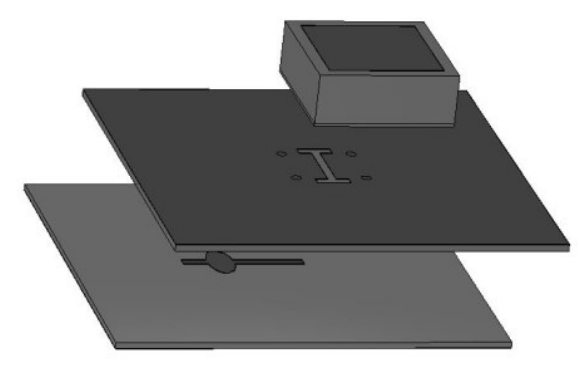

Fig. 1. Exploded view of $\mathrm{K}$ band microstrip antenna element.

The simulated S11 parameters of the single element in free space and in periodic environment are depicted on Fig. 2. The return loss bandwidth for $-10 \mathrm{~dB}$ is $500 \mathrm{MHz}$ for an antenna element in free space.

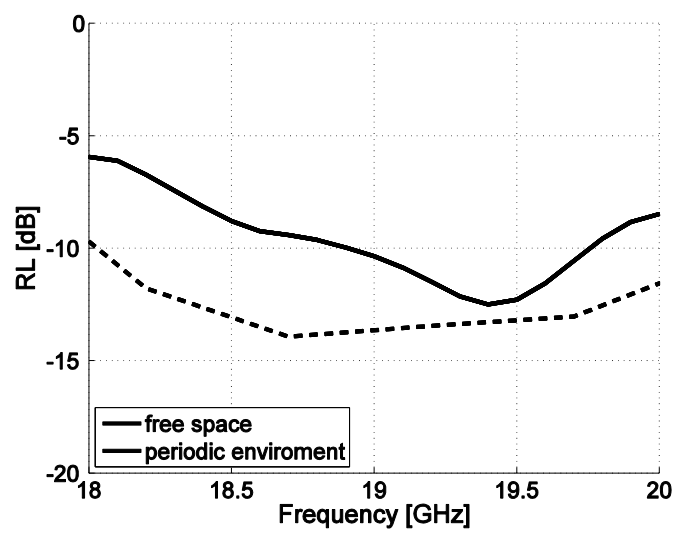

Fig. 2. Simulated S parameters of antenna elements.
The impedance bandwidth of the element is improved when using the antenna element in a large array (Fig. 2 periodic environment). The element was indeed optimized to operate in that environment. Decreasing the patch substrate height would also help in achieving a better impedance match. The optimum substrate thickness for this design is about $1.1 \mathrm{~mm}$ as shown in the simulations depicted in Fig. 3. The chosen substrate is available on the market with either 0.8 or $1.5 \mathrm{~mm}$ thickness but for large production a $1.1 \mathrm{~mm}$ thick substrate can be envisaged.

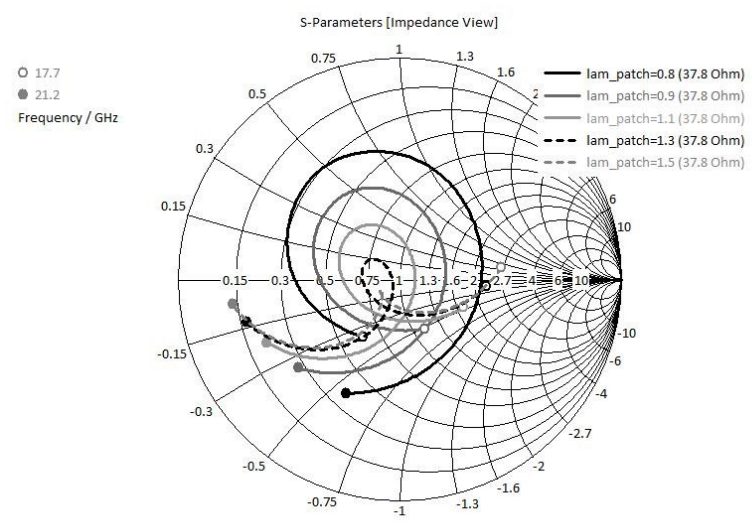

Fig. 3.Simulated return losses for different patch substrate thickness

\section{B. $M M I C$}

Only a few phase shifters and attenuators operating in $\mathrm{Ka}$ band, of digital and analog control, are available on the market. Digital control is preferable in our case to enable horizontal integration and to allow simpler communication with the control unit of the antenna. For this project the TGP2616 digital phase shifter and the TGL2615 attenuator, both from Triquint, were selected. The insertion losses of the components are $6.6 \mathrm{~dB}$ and $8.9 \mathrm{~dB}$, respectively - Fig. 4. To compensate these losses the UMS CHA3688 amplifier is placed with them in the chain.

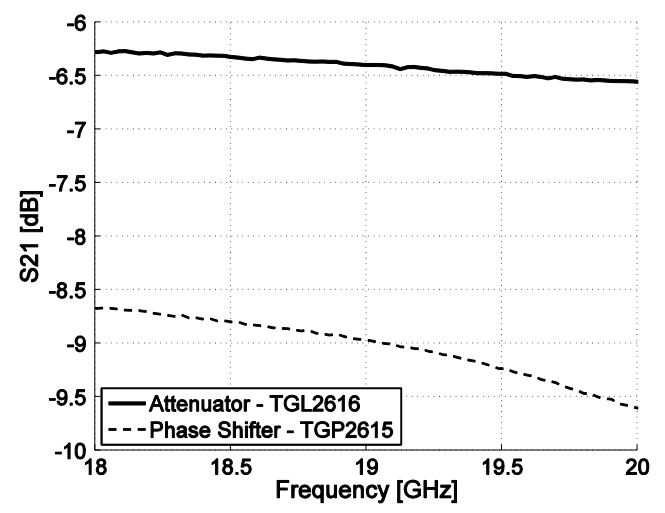

Fig. 4. Measured S21 parameters of TGL2616 attenuator and TGP2615 phase shifter for $0 \mathrm{~dB}$ attenuation and 0 degree phase shift.

\section{Antenna Array}

The proposed phase array allows two beams to be controlled independently. Each beam is created by 32 
element groups. Each group has one phase shifter, attenuator and amplifier as well as four antenna elements. The intermediate frequency (IF) input signals are upconverted and amplified and then distributed to the 32 antenna groups as depicted on Fig. 5. To accommodate all the control and DC lines with the RF beam forming network, a build-up with 6 copper layers has been designed.

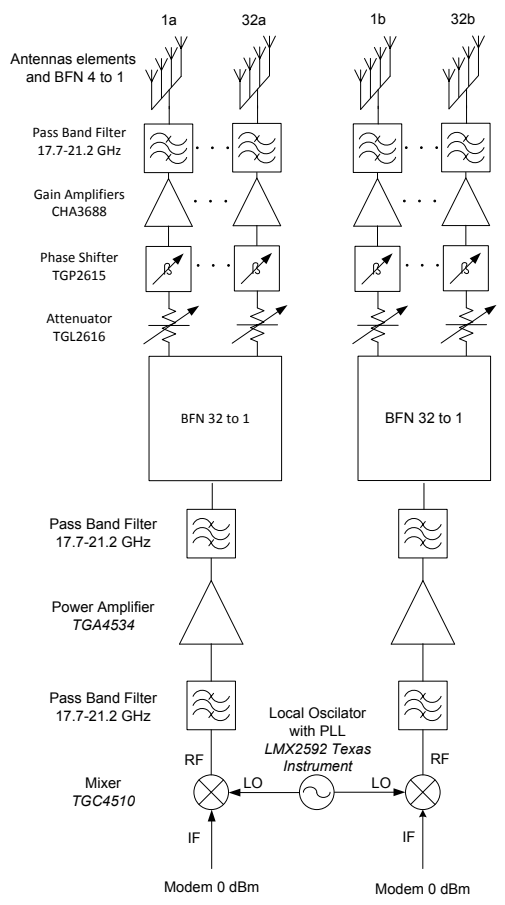

Fig. 5. Architecture diagram of the K band phase array.

The antenna array must satisfy an element density constraint to avoid main beam aliases (grating lobes). For a linear array (for scan in a single plane) this constraint is:

$$
d / \lambda<1 /(1+\sin \theta)
$$

According to the antenna specification and the previous equation the element grid was set to $8.5 \times 11.7 \mathrm{~mm}$. The radiation patterns for different scan angles of the antenna array consisting of 32 groups is depicted on Fig. 6. The directivity is higher than $25 \mathrm{~dB}$ even for 50 degree scan angle. The two beams may be combined at IF level to achieve even higher single-beam gain.

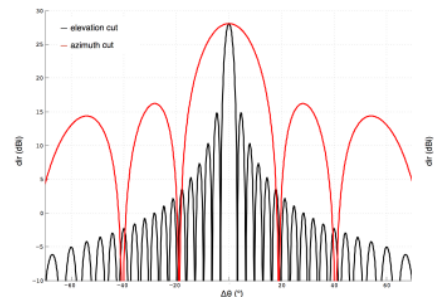

a)

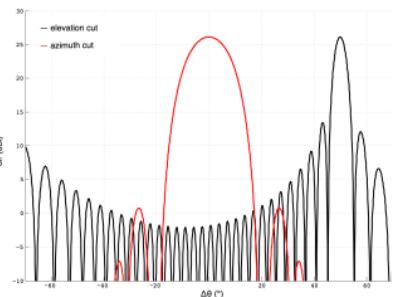

b)
Fig. 6. Simulated radiation pattern of developed phase array. a) Scan to $0^{\circ}$. b) Scan to $50^{\circ}$.
The first subarray has been manufactured and is under test. The final demo antenna array will be manufactured in December and measurements results will be presented in the final paper.

\section{CONCLUSIONS}

A novel concept of antenna array for future $5 \mathrm{G}$ backhaul networks and satellites communication has been presented. The antenna operates in $\mathrm{Ka}$ and has two independent beams which can be steered in one direction up to $50^{\circ}$. The presented solution uses low cost material to optimize a price and to make the antenna more attractive for customers. A further reduction of the cost will be possible when the custom MMIC that is being developed will enter high volume production.

\section{ACKNOWLEDGMENT}

The authors would like to acknowledgment the support form SANSA project.

\section{REFERENCES}

[1] SANSA, 'Shared Access Terrestrial-Satellite Backhaul Network enabled by Smart Antennas,' 2016, Available: http://www.sansah2020.eu, Accessed 01-Feb-2015.

[2] Bruce, E., and A. C. Beck, "Experiments with directivity steering for fading reduction," Proc. IRE, Vol. 23, No. 4, 357-371, Apr. 1935.

[3] I. Uchendu, and J. Kelly, "Survey of b steering techniques available for millimeter wave aplication," Progress in Electromagnetic Research B, vol. 68, pp. 35-54, May 2016.

[4] H. Kawakami, T. Ohira, "Electrically steerable passive array radiator (ESPAR) antennas," IEEE Antennas and Propagation Magazine vol. 47, Issue: 2, pp. 43-50, April 2005

[5] I. D. Robertson, S. Lucyszyn, "RFIC and MMIC Design and Technology," The Institute of Electrical Enginering 2009.

[6] David M. Pozar "A Review of Aperture Coupled Microstrip Antennas: History, Operation, Development, and Applications" Electrical and Computer Engineering University of Massachusetts at Amherst 